\title{
Estudo comparativo dos parâmetros cardiorrespiratórios e tempo de recuperação em cadelas submetidas a ovariosalpingohisterectomia e anestesiadas com halotano, isofluorano ou sevofluorano
}

\author{
Comparative study of clinical cardiorespiratory parameters and recovery times in bitches \\ submitted to ovariohysterectomy and anesthetized with halothane, isoflurane or sevoflurane
}

\author{
Ewaldo de MATTOS JUNIOR ${ }^{1}$; Kelly Cristiane ITO $^{1}$; Andreza CONTI-PATARA ${ }^{1}$; Haley Silva de \\ CARVALHO $^{1}$; Juliana de Araújo CALDEIRA ${ }^{1}$; Adriane REINOLDES ${ }^{1}$; Silvia Renata Gaido CORTOPASSI ${ }^{1}$
}

${ }^{1}$ Departamento de Cirurgia da Faculdade de Medicina Veterinária e Zootecnia da Universidade de São Paulo, São Paulo-SP

\begin{abstract}
Resumo
Objetivou-se comparar os efeitos cardiorrespiratórios, bem como sobre a recuperação em cadelas submetidas à ovariosalpingohisterectomia, e anestesiadas pelo halotano, isofluorano ou sevofluorano. Para tanto, utilizaram-se vinte e quatro cadelas sem raça definida, com peso médio de 15,03 e idade média de 3,93 anos, clinicamente saudáveis, avaliadas por meio de hemograma completo, contagem de plaquetas, bioquímica hepática e renal e teste de coagulação, sendo distribuídas em três grupos de oito animais. Todos os animais receberam acepromazina na dose de $0,1 \mathrm{mg} /$ $\mathrm{kg}$ i.m., como pré-tratamento e após 15 minutos a anestesia foi induzida com propofol na dose de $5 \mathrm{mg} / \mathrm{kg}$ i.v.. Para a manutenção utilizou-se halotano (grupo GAH), isofluorano (GAI) ou sevofluorano (GAS). Não houve diferença estatística entre os grupos em relação aos parâmetros cardiovasculares, observando redução discreta dos valores pressóricos após a indução, devido provavelmente a ação do propofol. A temperatura retal diminuiu gradativamente em função do tempo de anestesia, não apresentando diferença entre os grupos, porém GAI e GAS obtiveram valores inferiores dessa variável. Houve depressão respiratória nos três grupos, evidenciada pelo aumento da $\mathrm{PaCO}_{2}$ com consequente diminuição do $\mathrm{pH}$ arterial, porém sem diferença entre os grupos. O período obtido para extubação foi semelhante entre os grupos, no entanto, GAS apresentou menor tempo para posição quadrupedal comparado com GAH e GAI. A partir desses dados, conclui-se que diante dessa categoria animal e procedimento cirúrgico, o halotano, isofluorano e sevofluorano apresentaram características similares em relação aos parâmetros cardiovasculares, todos promoveram depressão respiratória e a recuperação foi mais rápida quando se optou pelo sevofluorano.
\end{abstract}

Palavras-chave: Anestesia. Halotano. Sevofluorano. Isofluorano. Cães.

\begin{abstract}
The cardiopulmonary effects and recovery times of halothane, isoflurane and sevoflurane were compared in bitches submitted to ovariohysterectomy. Twenty-four mongrel dogs were assigned in three groups of eight animals, with medium weight $15.03 \mathrm{~kg}$ and 3.93 years of age, designed groups GAH, GAI and GAS. All dogs received acepromazine $(0.1 \mathrm{mg} / \mathrm{kg}$, i.m.) as premedication and after 15 minutes, anesthesia was induced with propofol $(5.0 \mathrm{mg} / \mathrm{kg}$, i.v.) and maintenance anesthetics were halothane (GAH), isoflurane (GAI) and sevoflurane (GAS). No statistic difference was observed in cardiovascular parameters, but the SAP, DAP and MAP decreased slighty in moment M1 and this effect was associated with propofol. The rectal temperature decreased in function of the time of anesthesia, without difference among groups, but the AI and AS groups presented the lowest values of this parameter. Respiratory rate decreased in all groups, with an increase in the $\mathrm{PaCO}_{2}$ and a decrease in the $\mathrm{pH}$, without statistic differences. The extubation times were similar in the three groups. Time to standing was shorter in the AS group when compared with the GAH and GAI groups. On the basis of the results, for this animal category and surgical procedure halothane, isoflurane and sevoflurane were similar in cardiovascular parameters. All agents caused respiratory depression and the recovery times were shorter in sevofluorano group.
\end{abstract}

Keywords: Anesthesia. Halothane. Isoflurane. Sevoflurane. Dogs.

Correspondência para:

Ewaldo de Mattos Junior

Departamento de Cirurgia

Faculdade de Medicina Veterinária e Zootecnia

Universidade de São Paulo
Av. Prof. Orlando Marques de Paiva, 87, Cidade Universitária

CEP.: 05508-270. São Paulo, SP, Brasil

E-mail: ewaldomattos@hotmail.com

Recebido: 16/02/2009

Aprovado: 05/08/2010

Braz. J. Vet. Res. Anim. Sci., São Paulo, v. 47, n. 5, p. 403-412, 2010 


\section{Introdução}

Dentre as diversas modalidades anestésicas utilizadas na medicina veterinária, os agentes voláteis são os fármacos mais amplamente utilizados, para indução e manutenção anestésica. Isso se deve, principalmente, à possibilidade de alteração rápida nos planos anestésicos, eliminação em quase sua totalidade, independentes da via renal ou hepática, recuperação mais rápida, além de serem considerados seguros para as diversas categorias de risco ao qual o paciente se enquadra. Diferenças nas particularidades farmacológicas, como absorção, distribuição e eliminação, além de alterações nos diversos órgãos e sistemas, de cada agente determinam sua escolha para um determinado paciente ou procedimento ${ }^{1}$.

Os anestésicos inalatórios mais utilizados na medicina veterinária são o halotano, o isofluorano e o sevofluorano. O halotano promove alterações cardiovasculares mais intensas, reduzindo o débito cardíaco e a pressão arterial de maneira dose-dependente, sensibilizando o miocárdio à ação de catecolaminas, predispondo a arritmias cardíaca ${ }^{2}$ Eleva a frequência respiratória, porém, não é suficiente para manter o volume-minuto, resultando em aumento da pressão parcial de dióxido de carbono no sangue arterial $\left(\mathrm{PaCO}_{2}\right)$. O isofluorano, por sua vez, desencadeia efeitos cardiovasculares mínimos, mantendo o débito cardíaco, elevando a frequência cardíaca em concentração de até 2 CAM, podendo causar hipotensão $\operatorname{arterial}^{3}$. A depressão respiratória promovida por este agente é mais intensa que aquela causada pelo halotano, diminuindo o volume-minuto e consequentemente aumentando a $\mathrm{PaCO}_{2}$. O sevofluorano provoca taquicardia e hipotensão arterial no cão, ocorrendo também vasodilatação periférica e depressão da contratilidade miocárdica dose-dependente. A depressão respiratória causada por este agente é semelhante à do isofluorano ${ }^{4}$.

Sendo a ovariohisterecmia um procedimento realizado com frequência na rotina cirúrgica veterinária, o presente trabalho teve como objetivo avaliar as alterações cardiorrespiratórias e na recuperação pósoperatória imediata em cadelas submetidas a este procedimento cirúrgico e anestesiadas com halotano, isofluorano ou sevofluorano.

\section{Material e Método}

Este trabalho foi aprovado pelo Comitê de Bioética da Faculdade de Medicina Veterinária e Zootecnia da Universidade de São Paulo sob protocolo número 977/2006 e os proprietários consentiram na inclusão dos seus respectivos animais na pesquisa.

Foram utilizadas vinte e quatro cadelas, com peso médio de 15,09 $\pm 4,29 \mathrm{~kg}$, com idade média de 3,91 $\pm 1,23$ anos, clinicamente hígidas, encaminhadas ao Hospital Veterinário da Faculdade de Medicina Veterinária e Zootecnia da Universidade de São Paulo, para realização de ovariosalpingohisterectomia eletiva. Antes da ocorrência do procedimento, foram realizados exame clínico, avaliação hematológica constando de hemograma completo e contagem de plaquetas, além de perfil bioquímico hepático, renal e prova de coagulação sanguínea, excluindo-se do experimento qualquer animal que apresentasse alteração.

Após jejum sólido de 12 horas e hídrico de seis horas, os animais foram tratados com acepromazina (Acepran 0,2\%, Univet, Campinas, SP) na medicação pré-anestésica na dose de $0,1 \mathrm{mg} / \mathrm{kg}$ por via intramuscular e posteriormente foram distribuídos, de modo aleatório, em três grupos de oito animais. Decorridos 15 minutos, a via cefálica foi canulada com cateter de calibre apropriado e os animais receberam solução de Ringer com lactato (Solução de Ringer com Lactato de Sódio, Aster, Sorocaba, SP) no volume de $10 \mathrm{~mL} /$ $\mathrm{kg} /$ hora, durante todo o procedimento. A indução da anestesia foi realizada com propofol (Propovan 10 $\mathrm{mg} / \mathrm{mL}$, Cristália, São Paulo, SP), na dose de $5 \mathrm{mg} /$ $\mathrm{kg}$, lentamente por meio da via intravenosa (tempo de infusão de 60 segundos). Uma vez que os animais 
apresentaram relaxamento mandibular foi realizada a entubação orotraqueal com sonda de diâmetro apropriado, sendo em seguida conectada ao circuito valvular circular do aparelho de anestesia (Shogun, Takaoka, São Bernardo do Campo, SP), e mantidas em respiração espontânea com halotano (Tanohalo, Cristália, São Paulo, SP) (GAH), isofluorano (Isocris, Cristália, São Paulo, SP) (GAI) ou sevofluorano (Sevocris Cristália, São Paulo, SP ) (GAS), diluídos em oxigênio a $100 \%$.

O procedimento cirúrgico e anestésico, bem como a colheita de dados, foram realizados pelas mesmas pessoas. O estudo foi do tipo "cego" onde o responsável pela pesquisa não sabia qual o fármaco empregado na manutenção anestésica, além dos dados referentes à fração inspirada do fármaco em questão. O monitoramento da profundidade anestésica, bem como o ajuste das concentrações dos anestésicos inalatórios, foi baseada seguindo a escala preconizada por Guedel ${ }^{5}$.

Como analgesia pós-operatória foi empregado dipirona ( $25 \mathrm{mg} / \mathrm{kg}$ ) e cetoprofeno ( $2 \mathrm{mg} / \mathrm{kg})$ i.v., quando necessário o resgate analgésico foi realizado com tramadol $(2 \mathrm{mg} / \mathrm{kg})$ i.v.

Os parâmetros foram verificados e registrados em todos os grupos desde M0 até M6, sendo estes: M0 (basal), 15 minutos após a administração da medicação pré-anestésica; M1, 15 após a indução da anestesia e a intubação orotraqueal; M2, ligadura do pedículo ovariano; M3, sutura da musculatura; M4, sutura de pele e M5, dez minutos após o fim da administração do anestésico. A frequência (FC batimentos por minuto) e o ritmo cardíacos foram avaliados por meio da colocação dos eletrodos do eletrocardiógrafo (DX 2010, Dixtal, Manaus, AM) na pele dos membros dos animais. A pressão arterial média (PAM - mmHg) foi avaliada através do cateter introduzido na artéria femoral por via transdémica, conectado ao transdutor de pressão do monitor multiparamétrico (DX 2010, Dixtal, Manaus, AM).
A temperatura retal $\left(\right.$ Tretal $\left.-{ }^{\circ} \mathrm{C}\right)$ foi avaliada com auxílio de termômetro clínico inserido no reto dos animais.

A frequência respiratória ( $f$ - respirações por minuto), tensão de dióxido de carbono no final da expiração $\left(\mathrm{ETCO}_{2}-\mathrm{mmHg}\right)$, fração inspirada de oxigênio $\left(\mathrm{FiO}_{2}-\%\right)$ e fração expirada de anestésico (ETanest\%), foram obtidas por meio de analisador de gases (Datex Ultima, Helsinki, FI), do tipo aspirativo, sendo o sensor conectado entre a sonda endotraqueal e o circuito circular valvular do aparelho de anestesia. A saturação da oxi-hemoglobina periférica (\%) foi avaliada através do posicionamento do sensor do oxímetro (Datex Ultima, Helsinki, FI) na língua dos animais. O relaxamento muscular (RM) foi avaliado subjetivamente por meio da resistência à flexão de membros, da resistência à abertura da boca e da observação de atividade muscular não induzida, segundo o escore proposto a seguir: 3 = intenso (flacidez muscular total); 2 = regular (discreto tônus muscular); 1 = leve (importante tônus muscular), e $0=$ ausente (contrações durante manipulações).

Para fins de efeito comparativo com os dados descritos na literatura, foram calculados os valores da concentração alveolar mínima (CAM) dos respectivos agentes anestésicos dentro de cada momento nos grupos utilizando regra de três simples baseados no estudo de Frink Jr. et al. ${ }^{6}$.

A relação entre a pressão parcial de oxigênio e fração inspirada de oxigênio $\left(\mathrm{PaO}_{2} / \mathrm{FiO}_{2}\right)$ foi obtida através da divisão do valor de pressão parcial arterial de oxigênio pela fração inspirada de oxigênio.

Foram colhidas amostras de sangue arterial para

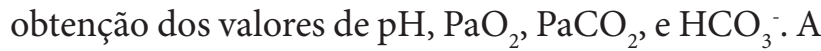
colheita de sangue arterial foi realizada pelo cateter de mensuração da pressão arterial direta, introduzido na artéria femoral. $\mathrm{O}$ exame foi realizado imediatamente após a colheita em analisador de $\mathrm{pH}$ e gases sanguíneos (Hemogasômetro ABL 5, Radiometer, Copenhaguem, DN). 
Os dados estão apresentados em forma de média, com respectivo desvio padrão. A análise estatística dos dados obtidos, para comparação entre momentos e grupos, foi realizada através de programa computacional Minitab versão 15, sendo aplicado do teste de normalidade de Kolmogorov-Smirnov, seguido de análise de variância e teste Tukey para os valores paramétricos e análise de Friedman e teste de KruskalWallis para os não paramétricos.

\section{Resultados}

O tempo total de anestesia compreendido do momento M0 a M5 foi para o grupo GAH: 63,25 \pm 9,85 minutos; GAI: 72,14 \pm 9,09 e GAS: 71,86 \pm 7,23.

Houve incremento da frequência cardíaca no grupo GH em M1 em relação ao valor basal ( $p>0,02)$. Em GI os momentos M1, M3, M4 e M5 foram superiores ao basal (p $<0,039, \mathrm{p}<0,037, \mathrm{p}<0,004$ e $\mathrm{p}<0,002$ respectivamente) e em GS M5 foi superior a M0 ( $\mathrm{p}<$ 0,009) (Tabela 1). Comparativamente entre os grupos houve diferença no momento M1 do grupo GH em relação à GI e GS (p < 0,05).

Em relação à pressão arterial média houve redução em GH no momento M1 ( $\mathrm{p}=0,001), \mathrm{M} 3(\mathrm{p}=0,003)$ e M4 ( $\mathrm{p}=0,006)$ comparado a M0, em GI e GS ocorreu no momento $\mathrm{M} 1$ ( $\mathrm{p}=0,013$ e $\mathrm{p}=0,015$ respectivamente). Entre os grupos não houve diferença ( $\mathrm{p}>$ 0,05) (Tabela 1).

A temperatura retal apresentou redução em todos os momentos e grupos comparativamente ao valor basal ( $\mathrm{p}<0,001)$, porém entre os grupos não houve diferença (Tabela 1).

As frações expiradas de anestésico e as concentrações alveolares mínimas não apresentaram diferença entre os momentos avaliados $(p>0,05)$ (Tabela 1).

Quanto ao relaxamento muscular, não houve diferença entre os momentos avaliados bem como entre grupos $(p>0,05)$ (Tabela 1).
Houve redução da frequência respiratória comparativamente no grupo GAH nos momentos M1, M2, M3 e M4, sendo inferiores ao valor basal ( $\mathrm{p}=$ $0,032, \mathrm{p}=0,016, \mathrm{p}=0,004$ e $\mathrm{p}=0,038$, respectivamente); no GAI foram nos momentos M1 ( $\mathrm{p}=$ $0,022), M 2(p=0,002), M 3(p=0,02)$ e M4 ( $p=$ $0,016)$ e em GAS no M1 ( $p=0,004), M 2(p=0,021)$ e M3 ( $p=0,041)$. Comparativamente entre os grupos GH apresentou valores superiores a GI e GS em M2 ( $\mathrm{p}=0,025)$ (Tabela 2).

No tocante ao $\mathrm{ETCO}_{2}$, houve redução dos valores do momento M5 em relação a M1 em GH e em GI (p $=0,002$ e $\mathrm{p}=0,003$ respectivamente). Em relação aos grupos, não ocorreu diferença significante $(\mathrm{p}>0,05)$ (Tabela 2). A saturação periférica de oxi-hemoglobina e a relação $\mathrm{PaO}_{2} / \mathrm{FiO}_{2}$ não apresentaram diferenças entre momentos ou grupos (Tabela 2). Os valores de $\mathrm{pH}, \mathrm{PaCO}_{2}$ e bicarbonato não apresentaram diferença estatística $(p>0,05)$ (Tabela 3).

O tempo para extubação não apresentou diferença entre os grupos ( $p>0,05)$, enquanto que o tempo para a posição quadrupedal foi inferior no grupo AS comparativamente aos grupos AH $(\mathrm{p}<0,01)$ e AI ( $\mathrm{p}$ $<0,05)$ (Figura 1).

\section{Discussão}

Todos os anestésicos voláteis causam alteração dose-dependente na performance cardiovascular. A magnitude dessas alterações, em algumas situações, está relacionada diretamente com outras variáveis que acompanham o procedimento anestésico, sendo eles: dose anestésica, tempo de anestesia, tratamento paralelo com outros fármacos, quantidade de $\mathrm{CO}_{2}$ circulante, ventilação mecânica e amplitude do estímulo nociceptivo ${ }^{1}$.

O halotano pode promover aumento na automaticidade do miocárdio e consequentemente propagação de impulsos provenientes de focos ectópicos, especialmente do ventrículo. Associado a isso, ocorre sensibi- 
Tabela 1 - Valores médios e \pm desvios padrão da frequência cardíaca (FC), pressão arterial média (PAM), temperatura retal (Tretal), fração expirada de anestésico (ETanest), concentração alveolar mínima (CAM) e relaxamento muscular (RM) em cadelas anestesiadas com halotano (GAH), isofluorano (GAI) ou sevofluorano (GAS) em diferentes momentos de avaliação - São Paulo - 2008

\begin{tabular}{|c|c|c|c|c|c|c|c|}
\hline Variável & Grupo & Mo & M1 & M2 & M3 & M4 & M5 \\
\hline \multirow{3}{*}{ FC (bpm) } & $\mathrm{GAH}$ & $107 \pm 22$ & $126 \pm 32^{*+}$ & $109 \pm 21$ & $106 \pm 20$ & $107 \pm 16$ & $129 \pm 24$ \\
\hline & GAI & $86 \pm 17$ & $103 \pm 20^{* \dagger}$ & $101 \pm 19$ & $108 \pm 18^{*}$ & $114 \pm 14^{*}$ & $133 \pm 23$ \\
\hline & GAS & $95 \pm 16$ & $95 \pm 24^{+}$ & $101 \pm 21$ & $114 \pm 18$ & $120 \pm 17$ & $139 \pm 22^{*}$ \\
\hline \multirow{3}{*}{ PAM (mmHg) } & $\mathrm{GAH}$ & $89 \pm 13$ & $62 \pm 6^{*}$ & $78 \pm 11$ & $62 \pm 13^{*}$ & $66 \pm 15^{*}$ & $82 \pm 18$ \\
\hline & GAI & $91 \pm 11$ & $66 \pm 12^{*}$ & $89 \pm 16$ & $80 \pm 19$ & $80 \pm 14$ & $100 \pm 19$ \\
\hline & GAS & $85 \pm 12$ & $65 \pm 14^{*}$ & $84 \pm 18$ & $75 \pm 16$ & $75 \pm 16$ & $104 \pm 27$ \\
\hline \multirow{3}{*}{ Tretal $\left({ }^{\circ} \mathrm{C}\right)$} & GAH & $38,6 \pm 0,22$ & $38 \pm 0,45^{\circ}$ & $37,8 \pm 0,5^{*}$ & $37,6 \pm 0,4^{*}$ & $37,3 \pm 0,4^{*}$ & $37,1 \pm 0,5^{\circ}$ \\
\hline & GAI & $38,5 \pm 0,41$ & $37,6 \pm 0,77^{*}$ & $37,3 \pm 0,8^{*}$ & $37,1 \pm 0,8^{*}$ & $36,9 \pm 0,9^{*}$ & $36,8 \pm 0,8^{*}$ \\
\hline & GAS & $38,6 \pm 0,63$ & $37,9 \pm 0,55^{*}$ & $37,4 \pm 0,9^{*}$ & $37 \pm 1,01^{*}$ & $36,8 \pm 1,0^{*}$ & $36,6 \pm 0,9^{*}$ \\
\hline \multirow{3}{*}{ ETanest (\%) } & GAH & - & $1,4 \pm 0,47$ & $1,5 \pm 0,50$ & $1,4 \pm 0,34$ & $1,2 \pm 0,25$ & $0,2 \pm 0,13$ \\
\hline & GAI- & - & $1,9 \pm 0,44$ & $2,4 \pm 0,63$ & $2,0 \pm 0,77$ & $2,0 \pm 0,53$ & $0,3 \pm 0,36$ \\
\hline & GAS & - & $1,2 \pm 0,19$ & $1,5 \pm 0,26$ & $1,5 \pm 0,31$ & $1,6 \pm 0,56$ & $0,5 \pm 0,91$ \\
\hline \multirow{3}{*}{ CAM } & GAH $\ddagger$ & - & $1,60 \pm 0,5$ & $1,66 \pm 0,5$ & $1,60 \pm 0,3$ & $1,30 \pm 0,3$ & $0,23 \pm 0,1$ \\
\hline & GAI $\ddagger$ & - & $1,46 \pm 0,4$ & $1,84 \pm 0,6$ & $1,53 \pm 0,7$ & $1,53 \pm 0,5$ & $0,23 \pm 0,4$ \\
\hline & GAS $\ddagger$ & - & $0,52 \pm 0,2$ & $0,66 \pm 0,3$ & $0,66 \pm 0,6$ & $0,70 \pm 0,6$ & $0,22 \pm 0,3$ \\
\hline \multirow{3}{*}{ RM } & GAH & - & $2 \pm 1$ & $2 \pm 1$ & $2 \pm 1$ & $2 \pm 1$ & $2 \pm 1$ \\
\hline & GAI & - & $1 \pm 1$ & $2 \pm 1$ & $2 \pm 0$ & $2 \pm 1$ & $2 \pm 0$ \\
\hline & GAS & - & $2 \pm 1$ & $2 \pm 1$ & $2 \pm 1$ & $2 \pm 1$ & $1 \pm 1$ \\
\hline
\end{tabular}

"Diferença em relação ao valor basal; ${ }^{\dagger}$ Diferença entre grupos; - Momento não avaliado; ‡ Baseado nos dados obtidos por Frink Jr et al. ${ }^{27}$.

Tabela 2 - Valores médios e \pm desvios padrão da frequência respiratória $(f)$, saturação periférica de oxigênio na hemoglobina $\left(\mathrm{SpO}_{2}\right)$, fração inspirada de oxigênio (\%), relação entre a parcial de oxigênio no sangue arterial e fração inspirada de oxigênio e pressão $\left(\mathrm{PaO}_{2} / \mathrm{FiO}_{2}\right)$ e tensão de dióxido de carbono no final da expiração $\left(\mathrm{ETCO}_{2}\right)$ em cadelas anestesiadas com halotano (GAH), isofluorano (GAI) ou sevofluorano (GAS) em diferentes momentos de avaliação - São Paulo - 2008

\begin{tabular}{|c|c|c|c|c|c|c|c|}
\hline \multirow[b]{2}{*}{ Variável } & \multirow[b]{2}{*}{ Grupo } & \multicolumn{6}{|c|}{ Momentos de avaliação } \\
\hline & & M0 & M1 & M2 & M3 & M4 & M5 \\
\hline \multirow{3}{*}{$f(\mathrm{mrm})$} & GAH & $25 \pm 6$ & $17 \pm 7^{*}$ & $17 \pm 7^{* \dagger}$ & $17 \pm 3^{*}$ & $18 \pm 5^{*}$ & $25 \pm 7$ \\
\hline & GAI & $24 \pm 6$ & $16 \pm 5^{*}$ & $10 \pm 5^{* \dagger}$ & $11 \pm 8^{*}$ & $11 \pm 6^{*}$ & $18 \pm 6$ \\
\hline & GAS & $19 \pm 8$ & $13 \pm 8^{*}$ & $11 \pm 2^{* \dagger}$ & $13 \pm 5^{*}$ & $16 \pm 5$ & $19 \pm 4$ \\
\hline \multirow{3}{*}{$\operatorname{ETCO}_{2}(\mathrm{mmHg})$} & GAH & - & $39 \pm 4$ & $39 \pm 4$ & $40 \pm 4$ & $38 \pm 4$ & $32 \pm 6^{*}$ \\
\hline & GAI & - & $38 \pm 4$ & $40 \pm 7$ & $38 \pm 6$ & $38 \pm 2$ & $31 \pm 3^{¥}$ \\
\hline & GAS & - & $41 \pm 9$ & $38 \pm 12$ & $40 \pm 9$ & $42 \pm 9$ & $38 \pm 7^{¥}$ \\
\hline \multirow{3}{*}{$\mathrm{SpO}_{2}(\%)$} & GAH & $99 \pm 1$ & $99 \pm 1$ & $98 \pm 2$ & $99 \pm 1$ & $98 \pm 2$ & $98 \pm 2$ \\
\hline & GAI & $99 \pm 1$ & $99 \pm 1$ & $99 \pm 1$ & $99 \pm 1$ & $98 \pm 1$ & $98 \pm 1$ \\
\hline & GAS & $99 \pm 1$ & $99 \pm 1$ & $99 \pm 1$ & $99 \pm 1$ & $99 \pm 1$ & $98 \pm 1$ \\
\hline \multirow{3}{*}{$\mathrm{PaO}_{2} / \mathrm{FiO}_{2}$} & GAH & - & $536 \pm 38$ & $531 \pm 31$ & $520 \pm 25$ & $531 \pm 24$ & $543 \pm 104$ \\
\hline & GAI & - & $514 \pm 38$ & $500 \pm 28$ & $504 \pm 19$ & $489 \pm 50$ & $593 \pm 89$ \\
\hline & GAS & - & $468 \pm 59$ & $431 \pm 65$ & $411 \pm 68$ & $391 \pm 70$ & $454 \pm 121$ \\
\hline
\end{tabular}




Tabela 3 - Valores médios e \pm desvios padrão do potencial hidrogeniônco no sangue arterial (pH), pressão parcial de dióxido de carbono no sangue arterial $\left(\mathrm{PaCO}_{2}\right)$, bicarbonato $\left(\mathrm{HCO}_{3}^{-}\right)$e relaxamento muscular $(\mathrm{RM})$ em cadelas anestesiadas com halotano $(\mathrm{GAH})$, isofluorano (GAI) ou sevofluorano (GAS) em diferentes momentos de avaliação - São Paulo - 2008

\begin{tabular}{|c|c|c|c|c|c|c|c|}
\hline \multirow[b]{2}{*}{ Variável } & \multirow[b]{2}{*}{ Grupo } & \multicolumn{6}{|c|}{ Momentos de avaliação } \\
\hline & & M0 & M1 & M2 & M3 & M4 & M5 \\
\hline \multirow{3}{*}{ pH } & GAH & - & $7,34 \pm 0,03$ & $7,32 \pm 0,04$ & $7,29 \pm 0,03$ & $7,31 \pm 0,03$ & $7,36 \pm 0,05$ \\
\hline & GAI & - & $7,31 \pm 0,03$ & $7,29 \pm 0,05$ & $7,30 \pm 0,04$ & $7,29 \pm 0,03$ & $7,37 \pm 0,06$ \\
\hline & GAS & - & $7,33 \pm 0,07$ & $7,30 \pm 0,08$ & $7,31 \pm 0,07$ & $7,30 \pm 0,04$ & $7,36 \pm 0,07$ \\
\hline \multirow{3}{*}{$\mathrm{PaO}_{2}(\mathrm{mmHg})$} & GAH & - & $527 \pm 50$ & $525 \pm 34$ & $516 \pm 24$ & $522 \pm 23$ & $110 \pm 23$ \\
\hline & GAI & - & $505 \pm 35$ & $494 \pm 29$ & $499 \pm 23$ & $482 \pm 53$ & $120 \pm 20$ \\
\hline & GAS & - & $444 \pm 48$ & $412 \pm 73$ & $395 \pm 83$ & $372 \pm 84$ & $147 \pm 131$ \\
\hline \multirow{3}{*}{$\mathrm{FiO}_{2}(\%)$} & GAH & & $98 \pm 2$ & $99 \pm 1$ & $99 \pm 1$ & $99 \pm 1$ & $20 \pm 0$ \\
\hline & GAI & - & $98 \pm 2$ & $99 \pm 1$ & $99 \pm 1$ & $99 \pm 1$ & $20 \pm 0$ \\
\hline & GAS & - & $95 \pm 4$ & $95 \pm 7$ & $95 \pm 8$ & $95 \pm 10$ & $21 \pm 0$ \\
\hline \multirow{3}{*}{$\begin{array}{c}\mathrm{PaCO}_{2} \\
(\mathrm{mmHg})\end{array}$} & GAH & - & $42 \pm 3$ & $44 \pm 5$ & $45 \pm 6$ & $43 \pm 4$ & $35 \pm 9$ \\
\hline & GAI & - & $44 \pm 4$ & $47 \pm 7$ & $44 \pm 5$ & $45 \pm 4$ & $35 \pm 4$ \\
\hline & GAS & - & $40 \pm 6$ & $46 \pm 9$ & $43 \pm 7$ & $42 \pm 10$ & $37 \pm 9$ \\
\hline \multirow{3}{*}{$\begin{array}{c}\mathrm{HCO}_{3} \\
(\mathrm{mmol} / \mathrm{L})\end{array}$} & GAH & - & $22 \pm 2$ & $22 \pm 1$ & $21 \pm 2$ & $21 \pm 1$ & $20 \pm 2$ \\
\hline & GAI & - & $21 \pm 1$ & $22 \pm 1$ & $21 \pm 1$ & $21 \pm 1$ & $20 \pm 2$ \\
\hline & GAS & - & $21 \pm 1$ & $22 \pm 1$ & $21 \pm 2$ & $20 \pm 4$ & $20 \pm 2$ \\
\hline
\end{tabular}

- Momento não avaliado

lização do miocárdio ou do seu sistema de condução à ação arritmogênica das catecolaminas endógenas e exógenas, predispondo o paciente a arritmias cardíacas $^{7}$, sendo descrito por Massone ${ }^{8}$ a sensibilização do miocárdio às catecolaminas quando se emprega o halotano, podendo resultar em fibrilação e assistolia.

Nesse estudo somente um animal que foi anestesiado com halotano apresentou arritmia cardíaca (12,5\%), caracterizada por batimentos ventriculares prematuros, ocorrendo treze por minuto, seguido de bradicardia $(\mathrm{FC}=48 \mathrm{bpm})$. O tratamento instituído foi sulfato de atropina, administrado lentamente na dose de 0,04 $\mathrm{mg} / \mathrm{kg}$, por via intravenosa, estabilizando a frequência e o ritmo após oito minutos da administração. Hikasa et al. ${ }^{9}$, Mutoh et al. ${ }^{10}$, Martin et al. ${ }^{11}$ e Polis et al. ${ }^{12}$ não relataram alterações no ritmo cardíaco nas pesquisas desenvolvidas utilizando halotano. A baixa porcentagem de arritmias associada ao halotano no presente estudo pode estar associada ao fato da administração prévia de acepromazina, pois Rezende et al. ${ }^{7}$ afirmam, em estudo feito com administração de adrenalina em cães anestesiados com halotano, que o emprego de acepromazina ou levomepromazina minimiza arritmias induzidas por esta catecolamina. Associado a isso, as idades dos animais, o tempo de procedimento relativamente curto e a não existência de comorbidades pode também ter influenciado em tal resultado.

A hipotensão arterial comumente ocorre durante e/ou imediatamente após a indução anestésica em bolus com propofol, sendo atribuída à diminuição da resistência vascular sistêmica ${ }^{13}$. Outro componente importante para essa alteração foi descrito por Puttrick et al. ${ }^{14}$ que observaram redução na pré-carga e na contratilidade cardíaca em cães que receberam propofol. Sem dúvida, tem-se também como fator potencializador, a acepromazina, que promove vasodilatação periférica e consequentemente hipotensão ${ }^{13}$. 


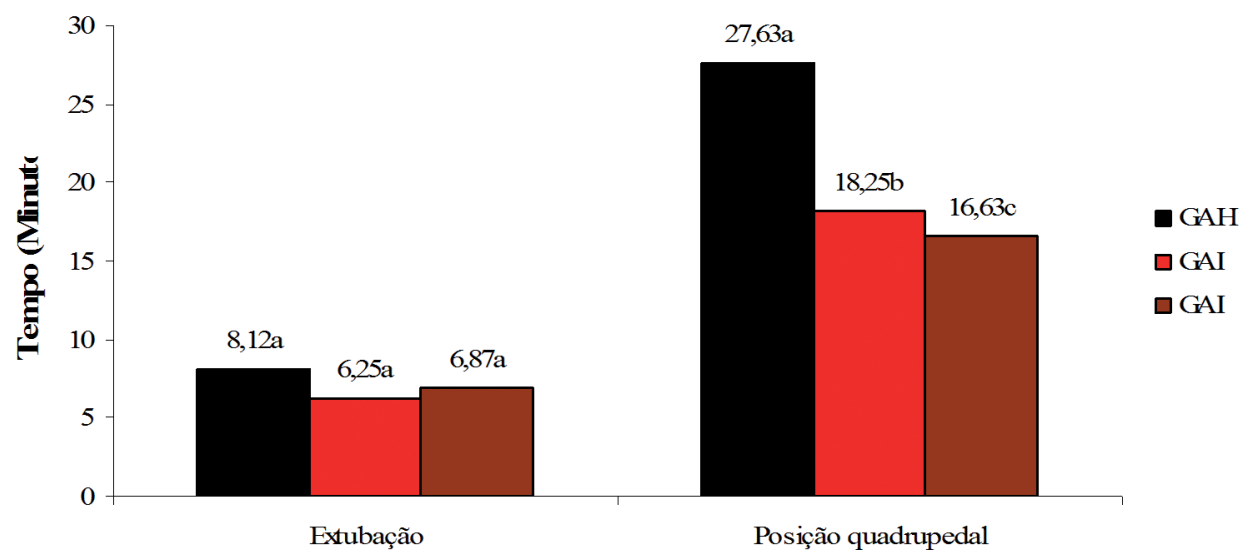

Figura 1 - Variação dos valores médios (minutos) do período de recuperação (extubação e posição quadrupedal). Letras minúsculas indicam igualdade ou diferença estatística entre grupos

A hipotensão arterial causada pelos agentes voláteis também é decorrente da diminuição da resistência vascular sistêmica ${ }^{15}$. Martin et al. ${ }^{11}$ verificaram valores superiores de halotano do que sevofluorano e isofluorano, e atribuíram esses resultados aos efeitos vasodilatadores mais intensos deflagrados pelo sevofluorano e isofluorano.

Os resultados encontrados no presente estudo são discordantes daqueles encontrados por Mutoh et al. ${ }^{10}$ que avaliaram concentrações de 1, 1,5 e 2 CAM de halotano, isofluorano e sevofluorano em cães, e verificaram diminuição de $\mathrm{PAD}$, PAS e PAM conforme aumento da concentração do anestésico. Nestas variáveis a redução foi contínua e diferente estatisticamente ao valor basal.

Os valores de temperatura retal apresentaram redução gradativa em todos os momentos em relação ao valor basal, embora sem apresentar diferença entre os grupos estudados. É importante ressaltar que os grupos AI e AS obtiveram valores inferiores em relação ao grupo AH em M4 e M5, com valores abaixo de 37 ${ }^{\circ} \mathrm{C}$. Os valores normais de temperatura para cães e gatos variam de $37,8-39,2{ }^{\circ} \mathrm{C}^{16}$, sendo considerada hipotermia valores de temperatura abaixo de $37^{\circ} \mathrm{C}^{15}$. Sabendo-se que o sevofluorano e isofluorano produzem vasodilatação mais intensa ${ }^{16}$, a perda de calor causada por estes agentes também poderá ser maior àquela causada pelo halotano. Sabe-se que a perda de calor é causada pela redistribuição para os compartimentos periféricos, sendo essa a principal causa de hipotermia induzida por anestésicos no homem ${ }^{17}$. O procedimento anestésico promove redistribuição de calor por dois mecanismos. O primeiro é a redução do limiar de temperatura necessário para causar vasoconstrição reflexa, em função da necessidade de baixas temperaturas para estimulação do centro termorregulador do hipotálamo. O segundo é a vasodilatação periférica causada diretamente pelos agentes anestésicos ${ }^{18}$.

A CAM do halotano, isofluorano e sevofluorano para o cão, segundo Steffey e Mama ${ }^{1}$ é de 0,86, 1,29 e 2,10 respectivamente. No presente estudo os valores ultrapassaram tais limites, chegando a 1,5 CAM para o halotano e 2,4 para o isofluorano. Valores mais baixos de halotano (média de 0,67) e isofluorano (média de 0,91) são relatados por Duke, Caulkett e Tataryn ${ }^{19}$ durante a realização de ovariohisterectomia, e experimentalmente por Polis et al. ${ }^{12}$ com valores médios de CAM de 0,89 e 1,39, para halotano e isofluorano, respectivamente. Sabe-se que os agentes administrados na medicação pré-anestésica podem influenciar nos parâmetros hemodinâmicos, bem como na concentração necessária de anestésico no intraoperatório. 
No presente estudo utilizou-se somente a acepromazina que comprovadamente produz apenas efeitos tranquilizantes ${ }^{20}$. Partindo desse pressuposto, supõese que os valores no presente trabalho se justifiquem pela não utilização de agentes adjuvantes no pré e no trans-operatório, que poderiam reduzir a concentração de tal fármaco pela promoção de analgesia suplementar, uma vez que os dados relatados por Martin et al. ${ }^{11}$, Polis et al. ${ }^{12}$ e Duke, Caulkett e Tataryn ${ }^{19}$ ao qual obtiveram CAM menores desses agentes, utilizaram romifidina, fentanil e hidromorfona na medicação pré-anestésica. O sevofluorano, por sua vez, mostrou-se mais eficiente em concentrações menores (média máxima de CAM de 1,6 em M4), fato observado também por Duke, Caulkett e Tataryn ${ }^{19}$ durante realização de ovariohisterectomia, no entanto utilizando hidromorfina como pré-tratamento. Martin et al. ${ }^{11}$ relataram concentração expirada de sevofluorano de $1,9 \%$ durante a realização de angiografia em cães. Como já citado anteriormente, tais valores de CAM podem ter sido influenciados pela medicação pré-anestésica, uma vez que nessas pesquisas foram utilizados fármacos com importante ação analgésica. Carrasco-Jimenez et al. ${ }^{21}$ utilizando cães tratados com acepromazina e buprenorfina e submetidos a diversos procedimentos cirúrgicos ortopédicos, utilizaram 1,25 CAM de sevofluorano em todos os animais, ressaltando que tal dose de anestésico foi suficiente para realização do procedimento cirúrgico, mantendo o nível de hipnose monitorado pelo índice biespectral adequado, não alterando também outros parâmetros hemodinâmicos que indicassem necessidade de maiores concentrações.

Não foram encontrados na literatura relatos com mesma metodologia que obtivesse valores de sevofluorano semelhantes ou inferiores; no entanto, como o valor de CAM refere-se à quantidade de anestésico para abolir a resposta dolorosa em $50 \%$ da população, supõe-se que os valores encontrados no presente trabalho, possam não ser incluídos nessa porcentagem da população, sendo necessária talvez, uma concentração menor de sevofluorano para esta categoria animal e tipo de procedimento.

Todos os anestésicos inalatórios causam depressão ventilatória dose-dependente ${ }^{10} \mathrm{com}$ diminuição da frequência respiratória resultando em hipoventilação com aumento na tensão de dióxido de carbono no final da expiração e na pressão parcial de dióxido de carbono no sangue arterial. Uma vez que essa substância possui propriedades acidificantes, há consequentemente, redução do potencial hidrogeniônico sanguíneo, gerando acidose respiratória, podendo levar a alterações orgânicas graves.

No presente estudo, a redução mais importante foi observada no grupo GAH, porém em todos os grupos a redução da frequência respiratória (embora dentro dos valores fisiológicos para espécie) resultou no incremento da $\mathrm{ETCO}_{2}$ e da $\mathrm{PaCO}_{2}$, apesar dos valores se preservarem dentro do padrão fisiológico [35 - 45 mmHg de acordo com Henão-Guerrero, Hodgson, e Mason $^{22}$. Vale ressaltar que essas variáveis, mesmo situando-se dentro do limite para a espécie, determinaram decréscimo progressivo do $\mathrm{pH}$ arterial abaixo dos valores normais. Mutoh et al. ${ }^{10}$ relataram resultados semelhantes em doses equipotentes de halotano, isofluorano e sevofluorano, e verificaram que foi o sevofluorano que causou maior redução da frequência respiratória e aumento da $\mathrm{PaCO}_{2}$ a 2 CAM, porém sem diferença estatística entre os grupos. Martin et al. ${ }^{11}$ e Polis et al. ${ }^{12}$ também observaram esse mesmo efeito sobre a FR e aumento da $\mathrm{ETCO}_{2}$, sendo em ordem crescente o sevofluorano, isofluorano e halotano. Estes dados discordam de Hikasa et al. ${ }^{9}$, que observaram valores elevados de $\mathrm{PaCO}_{2}$ e acidose respiratória em gatos que foram anestesiados com halotano comparativamente ao isofluorano e sevofluorano. Estes mesmos autores, em novo experimento com $\mathrm{cabras}^{23}$, relataram incremento da frequência respiratória sem diferença entre estes mesmos agentes, porém inadequados para manter níveis fisiológicos da $\mathrm{PaCO}_{2}^{23}$. 
Em cães, o halotano pode causar incremento da frequência respiratória, sendo insuficiente, entretanto para manter níveis adequados de $\mathrm{PaCO}_{2}^{24}$. O mesmo ocorre com o sevofluorano, sendo o isofluorano responsável pela depressão respiratória mais intensa dentre estes três agentes inalatórios em pacientes humanos $^{2}$. Estes efeitos devem-se principalmente a efeitos centrais, como depressão medular e também periférica como disfunção da musculatura intercostal ${ }^{24}$.

A velocidade de indução e recuperação é influenciada pelo coeficiente de partição sangue/gás ${ }^{25}$. O coeficiente de partição sangue/gás do sevofluorano, isofluorano e halotano, são respectivamente $0,68,1,46$ e 2,54', indicando recuperação anestésica mais rápida quando se opta pelo sevofluorano. Porém, fatores como fármacos empregados na medicação pré-anestésica e na indução, podem retardar o tempo de recuperação.

Neste estudo, a recuperação foi isenta de qualquer fenômeno que classificasse como insatisfatória nos três grupos. Não houve diferença entre os grupos quanto ao tempo para extubação, entretanto, o tempo para os animais assumirem a posição quadrupedal foi inferior no grupo AS em relação aos demais grupos. Estes resultados, porém, foram superiores àqueles encontrados em felinos por Hikasa et al. ${ }^{9}$ com valores de dois, três e quatro minutos para sevofluorano, isofluorano e halotano respectivamente, para extubação e sete, dez e 14 para posição quadrupedal. Cabe ressaltar que esses autores utilizaram somente os referidos agentes como indutores, sem qualquer pré-tratamento. Da mesma forma, Polis et al. ${ }^{12}$ em estudo com cães pré-tratados com droperidol e fentanil, verificaram valores de 9,46, 7,97 e 11,68 minutos com 2 CAM de sevofluorano, isofluorano e halotano, para extubação e concluíram que doses elevadas desses fár-

\section{Referências}

1. STEFFEY, E. P.; MAMA, K. Inhalation anesthetics. In: TRANQUILLI, W. J.; THURMON, J. C.; GRIMM, K. A. LUMB \& JONES' veterinary anesthesia and analgesia. 4. ed. Iowa: Blackwell Publishinhg, 2007. p. 355-394. macos prolongam o tempo de recuperação independente do coeficiente de partição sangue/gás e medicação pré-anestésica. A recuperação mais prolongada, discordante dos autores supracitados, deve-se provavelmente ao efeito da acepromazina, pois conforme os relatos de Cortopassi e Fantoni ${ }^{20}$, os efeitos clínicos deste podem persistir por até oito horas em cães. Assim sendo esse fármaco poderia ter prolongado o tempo de recuperação desses animais.

Martin et al. ${ }^{11}$ relataram como satisfatória a recuperação de cães submetidos a anestesia por estes mesmos três agentes voláteis por 30 minutos, não demonstrando diferença entre eles quanto à recuperação. Os valores obtidos foram de 15,8 minutos para halotano, 18,5 minutos para isofluorano e 17,9 minutos para sevofluorano e sugeriram que a semelhança entre os fármacos foi em função da romifidina ( $80 \mu \mathrm{g} / \mathrm{kg}$, i.m.) utilizada como medicação pré-anestésica, possuindo período hábil de até duas horas nessa dose ${ }^{26}$.

\section{Conclusões}

Diante dos dados apresentados acima, pode-se concluir que: os agentes halotano, isofluorano e sevofluorano promoveram incremento da frequência cardíaca e redução da pressão arterial média; os três fármacos causaram hipotermia, sendo mais pronunciada no grupo isofluorano e sevofluorano; os três agentes promoveram depressão ventilatória gerando acidose respiratória e o tempo para os animais assumirem posição quadrupedal foi inferior no grupo tratado com sevofluorano.

\section{Agradecimentos}

À FAPESP pelo suporte financeiro.
2. MORGAN, G. E.; MIKHAIL, M. S. Inhalational anesthestics. In: MORGAN, G. E.; MIKHAIL, M. S. Clinical anesthesiology. $2^{\text {nd }}$ ed. Stamford: Appleton \& Lange, 1996. p. 109-127. 
3. PRECKEL, B.; MÜLLENHEIM, J.; HOFF, J.; OBAL, D.; HEIDERHOFF, M.; THÄMER, V.; SCHLACK, W. Haemodynamic changes during halothane, sevoflurane and desflurane anaesthesia in dogs before and after the induction of severe heart failure. European Journal Anaesthology, v. 219, n. 10, p. 797-806, 2004.

4. BERNARD, J. M.; DOURSOUT, M. F.; WOUTERS, P.; HARTLEY, C. J.; MERIN, R. G.; CHELLY, J. E. Effects of sevoflurane and isoflurane on hepatic circulation in the chronically instrumented dog. Anesthesiology, v. 77, n. 3, p. 541-545, 1992.

5. GUEDEL, A. E. Inhalation anesthesia: a fundamental guide. New York: Macmillan, 1937. p. 25-28.

6. FRINK JR., E. J.; MORGAN, S. E.; COETZEE, A.; COENZEN, P. F.; BROWN JR., B. R. The effects of sevoflurane, halothane, enflurane, and isoflurane on hepatic blood flow and oxygenation in chronically instrumented greyhound dogs. Anesthesiology, v. 76, n. 1, p. 85-90, 1992.

7. REZENDE, M. L.; FARIAS, A.; BOLZAN, A. A.; FERREIRA, W. L.; LÉGA, E.; NUNES, N. Levomepromazina e acepromazina no bloqueio da arritmia induzida pela adrenalina em cães anestesiados pelo halotano. Ciência Rural, v. 32, n. 3, p. 433$438,2002$.

8. MASSONE, F. Anestesiologia veterinária: farmacologia e técnicas. 5. ed. Rio de Janeiro: Guanabara Koogan, 2008. 571 p.

9. HIKASA, Y.; KAWANABE, H.; TAKASE, K.; OGAWARA, $\mathrm{S}$. Comparisons of sevoflurane, isoflurane and halothane anesthesia in spontaneously breathing cats. Veterinary Surgery, v. 25, n. 3, p. 234-243, 1996

10.MUTOH, T.; NISHIMURA, R.; KIM, H.-Y.; MATSUNAGA, S.; SASAKI, N. Cardiopulmonary effects of sevoflurane, compared with halothane, enflurane and isoflurane, in dogs. American Journal Veterinary Research, v. 58, n. 8, p. 885$890,1997$.

11.MARTÍN, E.; REDONDO, J. I.; MOLLEDA, J. M.; SANTISTEBAN, J. M.; LÓPEZ, R.; GÓMEZVILLAMANDOS, R. Effects of sevoflurane, isoflurane and halothane anesthesia on fluorescein angiographic phases of dogs: a comparative study. Journal of Veterinary Medicine. A, Physiology, v. 48, n. 5, p. 267-275, 2001.

12.POLIS, I.; GASTHUYS, F.; VAN HAM, L.; LAEVENS, $\mathrm{H}$. Recovery times and evaluation of clinical hemodynamic parameters or sevoflurane, isoflurane and halothane in mongrel dogs. Journal of Veterinary Medicine. Animal Physiology, Pathology, Clinical Medicine, v. 48, n. 7, p. 401-411, 2001.

13. Almeida, E. M. P.; NUNES, N.; FANTINATTI, A. P.; SANTOS, S. P. S.; BOLZAN, A. A.; REZENDE, M. L. Efeitos cardiorrespiratórios da associação de tiletamina/zolazepam em cães (Canis familiaris) pré-tratados ou não pela acepromazina. Brazilian Journal Veterinary Research Animal Science, v. 37, n. 3, p. $210-215,2000$.

14.PUTTRICK, R. M.; DIEDERICKS, J.; SEAR, J. W.; FOËX, P.; RYDER, W. A.; GLEN, J. B. Effect of graded infusion rates of propofol on regional and global left ventricular function in the dog. British Journal Anesthesia, v. 69, n. 4, p. 375-381, 1992.

15.MATSUZAKI, Y.; MATSUKAWA, T.; OHKI, K.; YAMAMOTO, Y.; NAKAMURA, M.; OSHIBUCHI, T. Warming by resistive heating maintains perioperative normothermia as well as forced air heating. British Journal Anesthesia, v. 90, n. 5, p. 689-691, 2003.

16.BERNARD, J. M.; WOUTERS, P. F.; DOURSOUT, M. F.; FLORENCE, B.; CHELLY, J. E.; MERIN, R. G. Effects of sevoflurane and isoflurane on cardiac and coronary dynamics in chronically instrumented dogs. Anesthesiology, v. 72, n. 4, p. 659-662, 1990.

17.MATSUKAWA, T.; SESSLER, D. I.; SESSLER, A. M.; SCHROEDER, M.; OZAKI, M.; KURZ, A.; CHENG, C. Heat flow and distribution during induction of general anesthesia. Anesthesiology, v. 82, n. 3, p. 662-673, 1995.

18.ARMSTRONG, S. R.; ROBERTS, B. K.; ARONSOHN, M. Perioperative hypothermia. Journal Veterinary Emergency Critical Care, v. 15, n. 1, p. 32-37, 2005.

19.DUKE， T.; CAULKETT, N. A.; TATARYN, J. M. The effect of nitrous oxide on halothane, isoflurane and sevoflurane requirements in ventilated dogs undergoing ovariohysterectomy. Veterinary Anesthesia Analgesia, v. 33, n. 6, p. 343-350, 2006.

20.CORTOPASSI, S. R. G.; FANTONI D. T. Medicação préanestésica. In: FANTONI, D. T.; CORTOPASSI, S. R. G. Anestesia em cães e gatos. Roca: São Paulo, 2002. p. 151-164.

21.CARRASCO-JIMÉNEZ, M. S.; MARTÍN CANCHO, M. F.; LIMA, J. R.; CRISÓSTOMO, V.; USÓN-GARGALLO, J.; ESQUERRA, L. J. Relationships between a proprietary index, biespectral index, and hemodynamic variables as a means for evaluating depth of anesthesia in dogs with sevoflurane. American Journal Veterinary Research, v. 65, n. 8, p. 11281135,2004

22.HENÃO-GUERRERO, P. N.; HODGSON, D.; MASON, D. E. Capnography in veterinary medicine. Veterinary Anaesthesia Analgesia, v. 32, n. 4, p. 233-235, 2005.

23.HIKASA, Y.; OKUYAMA, K.; KAKUTA, T.; TAKASE, K.; OGASAWARA, S. Anesthetic potency and cardiopulmonary effects of sevoflurane in goats: comparison with isoflurane and halothane. Canadian Journal Veterinary Research, v. 62, n. 4, p. 299-306, 1998.

24.OLIVA, V. N. L. S. Anestesia Inalatória. In: FANTONI, D. T.; CORTOPASSI, S. R. G. Anestesia em cães e gatos. São Paulo: Roca, 2002. p. 174-183.

25.JOHNSON, R. A.; STRILLER, E.; SAWYER, D. C.; BRUNSON, D. B. Comparison of isoflurane with sevoflurane for anesthesia induction and recovery in adult dogs. American Journal Veterinary Research, v. 59, n. 4, p. 478-481, 1998.

26.LEMKE, K. A. Sedative effects of intramuscular administration of a low dose of romifidine in dogs. American Journal Veterinary Research, v. 60, n. 2, p. 162-168, 1999. 